\title{
Laboreal
}

Volume $12 \mathrm{~N}^{\circ} 1$ | 2016

Os Equipamentos de Proteção Individual (EPI):

protetores, mas nem sempre

\section{Análise da eficiência dos equipamentos de proteção aos agrotóxicos utilizados em saúde pública}

Análisis de la eficiencia de los equipos de protección para los plaguicidas utilizados en salud pública

Analyse de l'efficacité des équipements de protection contre les pesticides utilisés en santé publique

Efficiency analysis of protective equipment for pesticides used in public health

Marcelo Motta Veiga e Carlos Frederico Campelo de Albuquerque e Melo

\section{(2) OpenEdition}

\section{Journals}

Edição electrónica

URL: http://journals.openedition.org/laboreal/3232

DOI: $10.4000 /$ laboreal.3232

ISSN: 1646-5237

Editora

Universidade do Porto

Refêrencia eletrónica

Marcelo Motta Veiga e Carlos Frederico Campelo de Albuquerque e Melo, « Análise da eficiência dos equipamentos de proteção aos agrotóxicos utilizados em saúde pública », Laboreal [Online], Volume $12 N^{0} 1$ | 2016, posto online no dia 01 julho 2016, consultado o 08 outubro 2019. URL : http:// journals.openedition.org/laboreal/3232; DOI : 10.4000/laboreal.3232

Este documento foi criado de forma automática no dia 8 outubro 2019

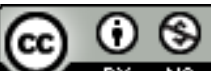

Laboreal está licenciado com uma Licença Creative Commons - Atribuição-NãoComercial 4.0 Internacional. 


\section{Análise da eficiência dos equipamentos de proteção aos agrotóxicos utilizados em saúde pública}

Análisis de la eficiencia de los equipos de protección para los plaguicidas utilizados en salud pública

Analyse de l'efficacité des équipements de protection contre les pesticides utilisés en santé publique

Efficiency analysis of protective equipment for pesticides used in public health

Marcelo Motta Veiga e Carlos Frederico Campelo de Albuquerque e Melo

\section{NOTA DO EDITOR}

http://dx.doi.org/10.15667/laborealxii0116mmv

Manuscrito recebido em: março/2016

Aceite após peritagem: maio/2016

\section{Os Agrotóxicos Utilizados em Saúde Pública}

1 O Brasil, devido a sua agricultura pujante, é o país onde se consome a maior quantidade de agrotóxicos no mundo (MMA, 2016). Muito embora a quase totalidade desses agrotóxicos sejam utilizados na agricultura, cresce sua utilização na Saúde Pública para controle de vetores e de hospedeiros intermediários de agentes causadores de doenças, e.g. dengue, zika, Chikungunya, febre amarela, malária, doença de Chagas, leishmaniose e esquistossomose (Abrasco, 2016). 
2 Os agrotóxicos utilizam centenas de substâncias químicas como princípios ativos. Os produtos comerciais devem ser registrados nos diferentes órgãos de acordo com suas utilizações aprovadas. A grande maioria dos agrotóxicos é registrada para o uso agrícola e apenas um pequeno número de agrotóxicos pode ser utilizado na Saúde Pública. Qualquer utilização de agrotóxicos no Brasil deve respeitar a legislação nacional vigente, independente de sua finalidade.

3 A Organização Mundial da Saúde define agrotóxico como toda "substância capaz de controlar uma praga que possa oferecer risco às populações ou ao meio ambiente". Neste sentido, os agrotóxicos são utilizados para controlar: bactérias, fungos, ervas daninhas, artrópodes, moluscos, roedores e quaisquer formas de vida danosas ao ambiente ou à saúde e bem-estar do homem (WHO, 2005).

4 Ressaltando que os agrotóxicos utilizados na área da Saúde Pública também devem estar registrados nos órgãos competentes e ter sua utilização aprovada para cada fim específico. No intuito de mitigar potenciais efeitos negativos, estes tiveram sua toxicidade e persistência reduzida ao longo dos anos. Atualmente, a maior parte dos agrotóxicos utilizados em Saúde Pública é do grupo químico dos organofosforados.

5 Contudo, devido a sua toxicidade e a sua ação biocida, a utilização da maioria dos agrotóxicos também está associada a enormes riscos e impactos à saúde humana e ao meio ambiente. Muito embora, a contaminação por agrotóxicos possa ocorrer de várias formas, os maiores riscos estão associados ao processo de trabalho.

\section{A Contaminação por Agrotóxicos}

6 A Organização Internacional do Trabalho (OIT) estabelece que os riscos à segurança e à saúde dos trabalhadores devem ser identificados e avaliados de forma contínua e que as medidas de prevenção e proteção devem ser implementadas na seguinte ordem de prioridades (OIT, 2001): eliminar os riscos; controlar a geração do risco; minimizar os riscos; e onde houver riscos residuais que não puderem ser evitados ou controlados por medidas coletivas, deve-se utilizar Equipamento de Proteção Individual (EPI).

7 O uso de Equipamento de Proteção Individual (EPI) é uma solução considerada inadequada nos projetos de Saúde e Segurança no Trabalho (SST). No entanto, dentro de uma visão integrada e sistêmica de abordagem dos problemas ocupacionais, os equipamentos de proteção devem ser considerados como tecnologias complementares de proteção. A eficiência de todo sistema de SST está intimamente relacionada à forma como é conduzida e balanceada, no processo decisório, a escolha das alternativas de prevenção, proteção e controle (OIT, 2001).

8 Um projeto eficiente de SST deve contemplar, num enfoque sistêmico, a integração de todos os elementos relevantes para estabelecer políticas e estratégias adequadas a cada realidade situacional.

9 A utilização de EPI faz parte da realidade de muitos trabalhadores. Muitas atividades profissionais envolvem manipulação de agrotóxicos, onde o controle dos riscos não pode ser exercido na fonte. Esses trabalhadores são expostos a riscos que devem ser controlados por todos os meios técnicos de segurança possíveis. Nesses aspectos, se intensifica a importância do emprego de meios individuais de proteção, os quais devem ter critérios de indicação definidos para cada ambiente e situação de trabalho. 

contaminação dos trabalhadores, podendo resultar em danos à saúde. (Blair et al., 2005). Verifica-se que em condições reais de trabalho, $99 \%$ da contaminação por agrotóxicos ocorre por via dérmica e apenas $1 \%$ ocorreria por via respiratória (Van Hemmen, 1992; Machera, Goumenou \& Glass, 2003).

11 Diversos autores estudaram a eficiência dos equipamentos de proteção individual associado ao risco de exposição aos agrotóxicos pelos trabalhadores (Tácio, Oliveira \& Machado-Neto, 2008). Machera, Tsakirakis, Charistou, Anastasiadou e Glass (2009) afirmam que o design das roupas de proteção e o tipo de costura influenciam significativamente a permeabilidade.

Este estudo analisou a eficiência dos equipamentos de proteção individuais à permeação dos agrotóxicos utilizados por profissionais de saúde pública no combate ao mosquito Aedes aegypti, vetor de diversas doenças (e.g. Dengue, Zika, Febre Amarela e Chikungunya). A permeabilidade foi analisada através do método da pipeta, calculandose as porcentagens de repelência, de retenção e de penetração de agrotóxicos nos materiais usados na fabricação dos EPIs.

\section{A Eficiência dos Equipamentos de Proteção}

13 Os equipamentos de proteção individual (EPI) são usados para mitigar as exposições dérmicas e respiratórias dos trabalhadores que pulverizam agrotóxicos. Atuam por repelência ou por impermeabilidade, e reduzem o contato direto das substâncias com os trabalhadores (Lee \& Obendorf, 2006).

A permeabilidade é o processo pelo qual o produto químico move-se pelo material de proteção em nível molecular. As moléculas do produto químico em contato com a superfície do material são absorvidas, em seguida ocorre à difusão e dispersão dessas moléculas pelo lado interno da superfície do material (Shaw, Cohen \& Hinz, 2004).

Os EPI podem ser confeccionados com materiais impermeáveis não porosos (e.g. filmes plásticos e borracha) ou com materiais porosos (e.g. tecidos de algodão e mistos de algodão e poliéster). Materiais impermeáveis seriam naturalmente protegidos contra a penetração. Porém, não se deve confundir a permeabilidade do material com a permeabilidade do EPI. A permeabilidade do EPI não se dá somente através do material, mas também devido ao design. A penetração dos agrotóxicos nos equipamentos de proteção individual ocorre por meio da passagem dos agrotóxicos pelos poros, emendas e costuras (Shaw, Cohen, Hinz \& Herzig, 2001).

16 Materiais porosos resistem à penetração pela repelência ou retenção dos agrotóxicos. Dessa forma, a repelência, retenção e ou a penetração são características usadas para medir a eficiência de materiais porosos das vestimentas de proteção contra os agrotóxicos (Shaw et al., 2001).

17 Propriedades estruturais dos tecidos como distribuição geométrica das fibras, espessura, gramatura, composição das fibras são importantes barreiras contra penetração aos agrotóxicos, pois influenciam na porosidade dos tecidos. Muitos materiais porosos são recobertos com impermeabilizantes, que aumentam a repelência. Esses fatores devem ser considerados na seleção dos materiais de proteção e na construção de equipamentos de proteção (Lee \& Obendorf, 2006). 
18 Alguns fatores podem interferir na permeabilidade dos agrotóxicos em materiais impermeáveis são: permeabilidade do ar $(\mathrm{mm} / \mathrm{s})$, taxa de transmissão de vapor de água $\left(\mathrm{g} / \mathrm{h} \cdot \mathrm{m}^{2}\right)$, viscosidade $(\eta)$, tensão superficial $(\mathrm{mN} / \mathrm{m})$ dos agrotóxicos e características do material de proteção (Zhang \& Raheel, 2003; Jain \& Raheel, 2003).

19 Meirelles, Veiga e Duarte (2012) consideram uma "falha de projeto" a permeabilidade dos Equipamentos de Proteção Individuais. Ademais, verifica-se que todos os testes laboratoriais somente são feitos em EPIs novos. Há carência de informação sobre a redução da proteção em decorrêncisabelbferreira@hotmail.comia do desgaste do material do EPI por utilização e por lavagens (Veiga, 2007).

Baldi et al. (2006) constataram que além da ineficácia dos EPIs devido a permeabilidade, há um aumento do risco de contaminação dos trabalhadores. A contaminação do trabalhador não se dá somente durante a exposição direta ao agrotóxico, mas, também, ao vestir e ao despir os EPIs usados impregnados com agrotóxicos por retenção.

21 Ressalta-se que no Brasil, somente a partir de 2010, o nível de proteção dos EPI para o controle da exposição dérmica foi regulamentado, passando a ser exigido na aprovação de novos EPI. Antes de 2010, os EPI eram aprovados sem passar por testes de avaliação do nível de permeação a produtos químicos, e.g. aos agrotóxicos.

\section{A Regulamentação dos Equipamentos de Proteção Individual (EPI)}

22 Equipamento de Proteção Individual (EPI) é definido pela Norma Regulamentadora $n^{\circ} 6$, aprovada pela Portaria no 3.214/78, do Ministério do Trabalho, como: "todo dispositivo ou produto, de uso individual utilizado pelo trabalhador, destinado à proteção de riscos suscetíveis de ameaçar a segurança e a saúde no trabalho".

23 Todo EPI deve possuir um Certificado de Aprovação (CA) expedido pelo Ministério do Trabalho. Este certificado identifica que o equipamento passou por um processo de registro junto ao órgão controlador. Nesse processo, o fabricante ou importador fornece ao órgão registrante, dentre outros documentos, um memorial descritivo do EPI, incluindo, no mínimo, as suas características técnicas principais, os materiais empregados na sua fabricação e o uso a que se destina.

24 A partir de julho de 2010 com a publicação da Portaria 189 e posteriormente com a Portaria 246 de 29 de junho de 2011, passou-se a exigir também um laudo de ensaio do EPI emitido por laboratório devidamente credenciado pelo Ministério do Trabalho.

Os ensaios que dão origem aos laudos seguem normas técnicas nacionais e internacionais, estabelecidas pela ISO/DIS27065:2010 e visam simular, em condições de laboratório, as situações de uso dos equipamentos novos, medindo assim sua capacidade de controle dos riscos a que se propõem a controlar.

26 A partir da análise e da aprovação do registro do equipamento, este passa a ter um número de $\mathrm{CA}$, o qual, juntamente com o nome da empresa fabricante ou importadora, deverá estar presente no equipamento em caracteres que não se apaguem e estejam em local bem visível.

27 A Portaria 121, de 30 de setembro de 2009, define os requisitos obrigatórios e as normas técnicas aplicáveis aos EPI. A partir da publicação da Portaria 189, de julho de 2010, foram estabelecidos os níveis de proteção dos EPI para o controle da exposição dérmica 
de acordo com órgãos de padronização internacional como a ISO (International Organization for Standardization) que publicou a ISO/DIS 27065:2010. Estabeleceu-se a obrigatoriedade do atendimento mínimo ser o descrito no nível de desempenho $1 \mathrm{~b}$, o qual define que a penetração da substância teste no material para confeccionar os EPI deve ser menor ou igual a 40\%, utilizando-se o procedimento da norma ISO 22608:2004.

Em 29 de junho de 2011, a Portaria 246 alterou a Portaria 189 e estabeleceu que os EPI devem, no mínimo, atender ao descrito no nível de desempenho 2 da norma ISO 27065. O nível de desempenho 2 determina o limite da porcentagem de penetração, deva ser menor ou igual a 5\%, pelo procedimento da norma ISO 22608:2004. A ISO 22608 define a mensuração da repelência, retenção e penetração em equipamentos de proteção aos agrotóxicos, além de descrever o método da pipeta, gravimetria.

\section{Material e Métodos}

Nesse estudo, todas as análises da permeabilidade dos materiais nos EPI selecionadas foram realizadas em um laboratório credenciado pelo Ministério do Trabalho para análises de EPIs visando à obtenção de Certificados de Aprovação seguindo normas internacionais padronizadas.

Devido o seu amplo uso, simplicidade e reconhecimento, o método da pipeta, algumas vezes chamado de método gravimétrico, é o mais utilizado para avaliar a repelência, a retenção e a penetração dos agrotóxicos nos materiais hidro-repelentes utilizados na confecção dos EPI (Raheel, 1988; Shaw \& Hill, 1991; Shaw et al., 2001).

Este método foi revisado e ampliado por órgãos de padronização internacional como a ISO e a ASTM, dando origem as normas ISO 22608 e ASTM 2130.

Essas normas padronizam os procedimentos para avaliar a repelência, a retenção, e a penetração de um volume conhecido de agrotóxico líquido aplicado em um determinado material (EPI). Volumes de 0,1 $\mathrm{ml}$ e de $0,2 \mathrm{ml}$ de agrotóxico são aplicados sobre o material dos EPI, simulando um baixo e um alto nível de exposição dos trabalhadores.

O método da pipeta foi utilizado por Raheel (1988) em estudo de mensuração de eficiência de treze tecidos porosos e não porosos ao inseticida carbaryl e ao herbicida atrazine. Neste estudo, verificou-se que houve uma proteção próxima de $100 \%$ dos tecidos tratados com hidrorrepelentes $\left(\right.$ Tyvek $^{\circledR}$ ); tecido de composição $100 \%$ algodão; tecido de composição 65/35 algodão/poliéster e tecido de composição 50/50 algodão/ poliéster.

34 Machera et al. (2009) utilizando o método da pipeta testou tecidos de algodão hidrófilo, algodão repelente e algodão com revestimento de poliéster repelente e detectaram uma proteção 5,5 vezes maior em tecidos com tratamento repelente a água em comparação aos tecidos de algodão simples.

Entretanto, o método da pipeta não é indicado para avaliar agrotóxicos voláteis, pois o tempo de aplicação necessário para análise seria suficiente para ter perdas do material, comprometendo a confiabilidade do resultado.

Nesse estudo, os seguintes materiais foram utilizados: micropipeta; lâminas de papéis de filtro; balança com precisão de 0,0001 g; placa de acrílico de 10 x $10 \mathrm{~cm}$, utilizada 
como base; lâmina de papel absorvente Whatman Benchkote Plus com dimensões de $8 \mathrm{x}$ $8 \mathrm{~cm}$; glifosato; cronômetro; pinça metálica.

\section{Seleção do EPI}

Este estudo selecionou os equipamentos de proteção utilizados nas atividades de controle espacial do mosquito Aedes aegypti. Estes EPIs eram efetivamente utilizados por trabalhadores da equipe de controle de vetores de uma Secretaria Municipal de Saúde no combate a vetores através de pulverização de agrotóxicos. A fim de produzir resultados comparáveis, todos os EPIs analisados possuíam o mesmo Certificado de Aprovação (CA). Os EPIs testados foram coletados aleatoriamente dentre os EPIs novos (sem uso) disponíveis no estoque da Secretaria Municipal de Saúde.

No intuito de padronizar as análises, as amostras de tecidos de todos os EPIs foram retiradas nos mesmos locais previamente selecionados por conveniência de forma a reproduzir as mesmas áreas de exposição nos EPIs. Assumiu-se que por se tratarem de EPIs novos, o local de seleção das amostras de tecidos não influenciaria nos resultados, o que não seria o caso se as análises fossem feitas em EPIs usados. Por isso, não houve maiores preocupações com os locais de retiradas das amostras do tecido sem costura, com costura simples e com costura rebatida. A Figura 1 mostra os locais em um EPI na qual foram retiradas amostras para análise e a Figura 2 apresenta amostras dos tecidos sem costura, com costura simples e com costura rebatida, respectivamente.

Figura 1. Peça de EPI identificando os locais de retirada das amostras de tecido para análise.

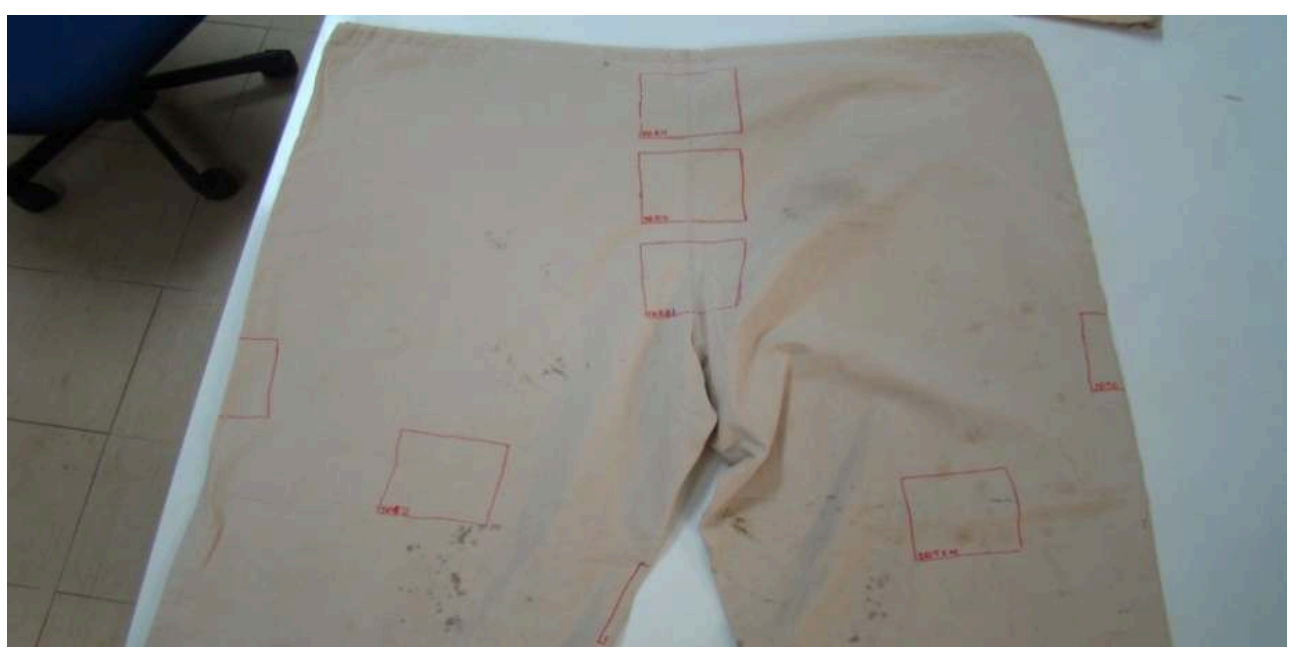


Figura 2. Amostras do tecido a) sem costura, b) costura simples e c) costura rebatida.

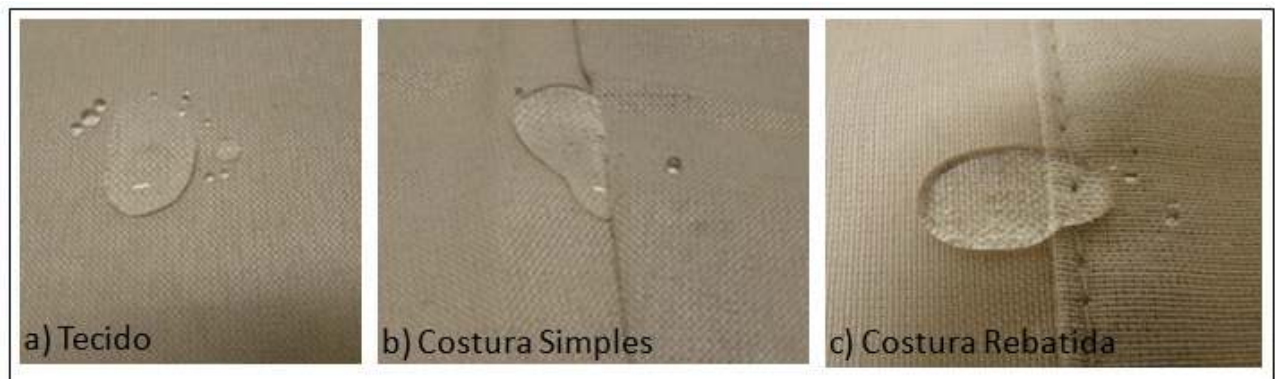

Espera-se que a penetração seja maior nos tecidos com costura e que seja afetada pelo tipo de costura, devido aos diferentes ordenamentos do tecido.

\subsection{Descrição do teste de repelência, retenção e penetração.}

Para a realização do teste de repelência, retenção e penetração foi utilizado o procedimento da gravimetria descrito nas normas ISO 22608 e ASTM F 2130, que são similares. Este teste é utilizado para avaliar a eficiência de materiais de proteção a produtos químicos tóxicos por meio da gota dispensada com uma micropipeta.

41 A Figura 3 ilustra de forma esquemática a montagem para realização do procedimento de gravimetria para avaliação de eficiência de materiais por meio da gota dispensada com micropipeta.

Figura 3. Esquema de montagem do teste de avaliação de materiais repelentes, de acordo com as normas ISO 22608 e ASTM F2130.

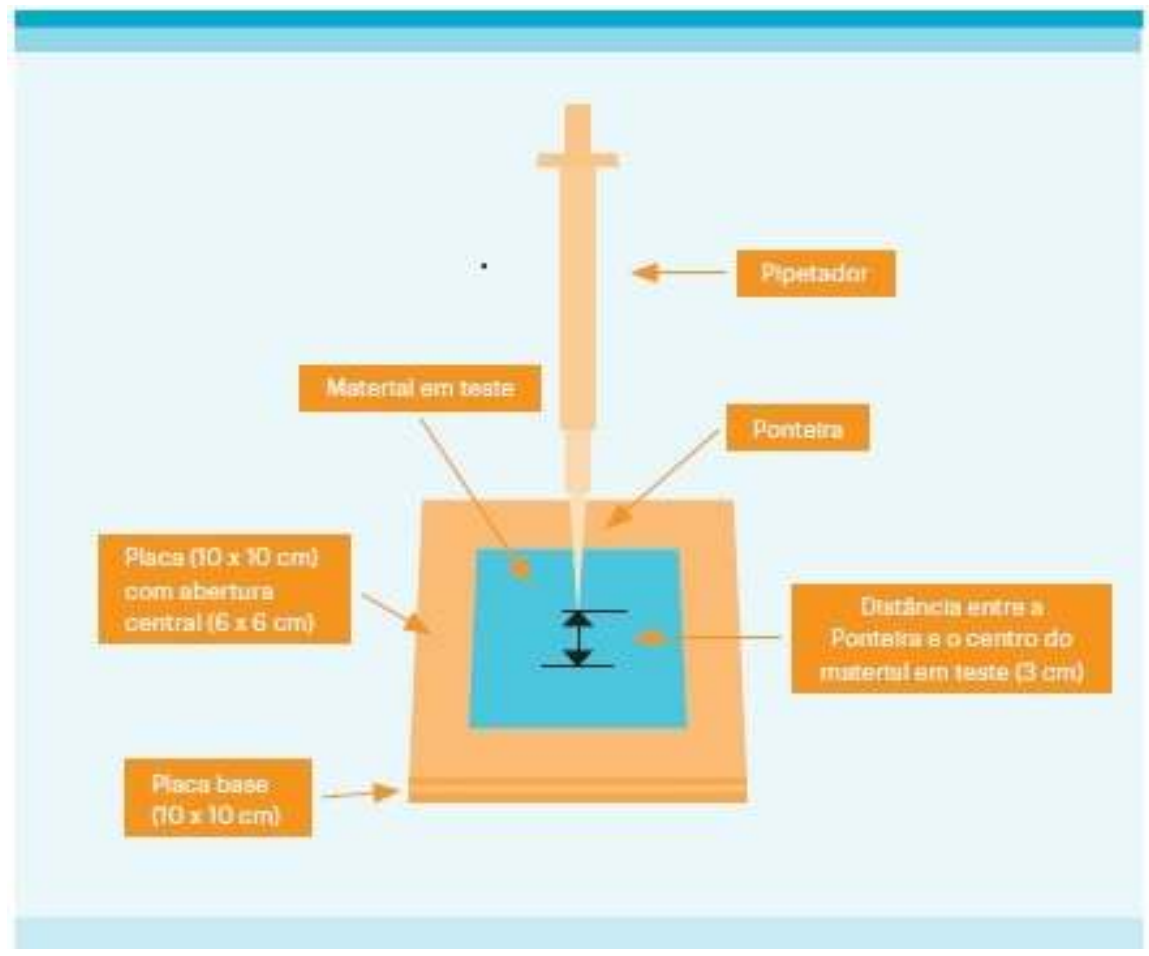

O procedimento utilizado para a contaminação das amostras e acondicionamento dos materiais foi realizado estritamente segundo as referidas normas. Considerou-se como 
amostra cada recorte de tecido dos EPI. Foram analisadas dez amostras de tecido para cada tipo de material (Tecido Simples; Costura Simples; e Costura Rebatida), perfazendo um total de 30 amostras. Nessas 30 amostras foram medidas a repelência, a retenção e a penetração, perfazendo um total de 90 resultados. O procedimento utilizado para a contaminação das amostras dos materiais está descrito a seguir:

1 - Acondicionamento das amostras dos materiais e das lâminas dos papéis de filtro por 24 horas antes do teste.

2 - Pesagem, em balança com precisão de 0,0001 $\mathrm{g}$ das amostras dos materiais e das laminas dos papéis de filtro imediatamente antes do início dos testes.

3 - Sobre uma placa de acrílico de $10 \times 10 \mathrm{~cm}$, utilizada como base, foram colocadas uma lâmina de papel absorvente Whatman Benchkote Plus com dimensões de $8 \times 8 \mathrm{~cm}$. Este papel tem uma face impermeabilizada com um filme de polietileno, que é colocada para baixo e voltada para a placa base.

4 - Sobre a lâmina de papel, com a face absorvente voltada para cima, foi colocada uma amostra do material de proteção em teste, com dimensões de $8 \times 8 \mathrm{~cm}$. Outra placa de acrílico de $10 \times 10 \mathrm{~cm}$ e com uma abertura central quadrada de $6 \times 6 \mathrm{~cm}$, usada como uma moldura, foi colocada sobre a amostra do material de proteção em teste. A placa moldura foi utilizada para manter e uniformizar o contato entre a superfície do material em teste com o papel absorvente.

5 - Depositou-se uma gota de $0,2 \mathrm{ml}$ de glifosato, com densidade de 49 dynas e viscosidade de $1,18 \mathrm{cP}$, no centro da amostra dos materiais com uma micropipeta e, ao mesmo tempo, disparou-se um cronômetro. Depois de 10 minutos, retirou-se a placa moldura e foi colocada a segunda lâmina do papel absorvente sobre o material em teste com a face impermeável voltada para cima (Figura 4). Em seguida, recolocou-se a placa moldura sobre a segunda lâmina de papel absorvente. Depois de dois minutos, desmontou-se o teste na sequência inversa de montagem com o auxílio de pinça metálica. 
Figura 4. Amostra com gota de $0,2 \mathrm{ml}$ de glifosato depositada com micropipeta.

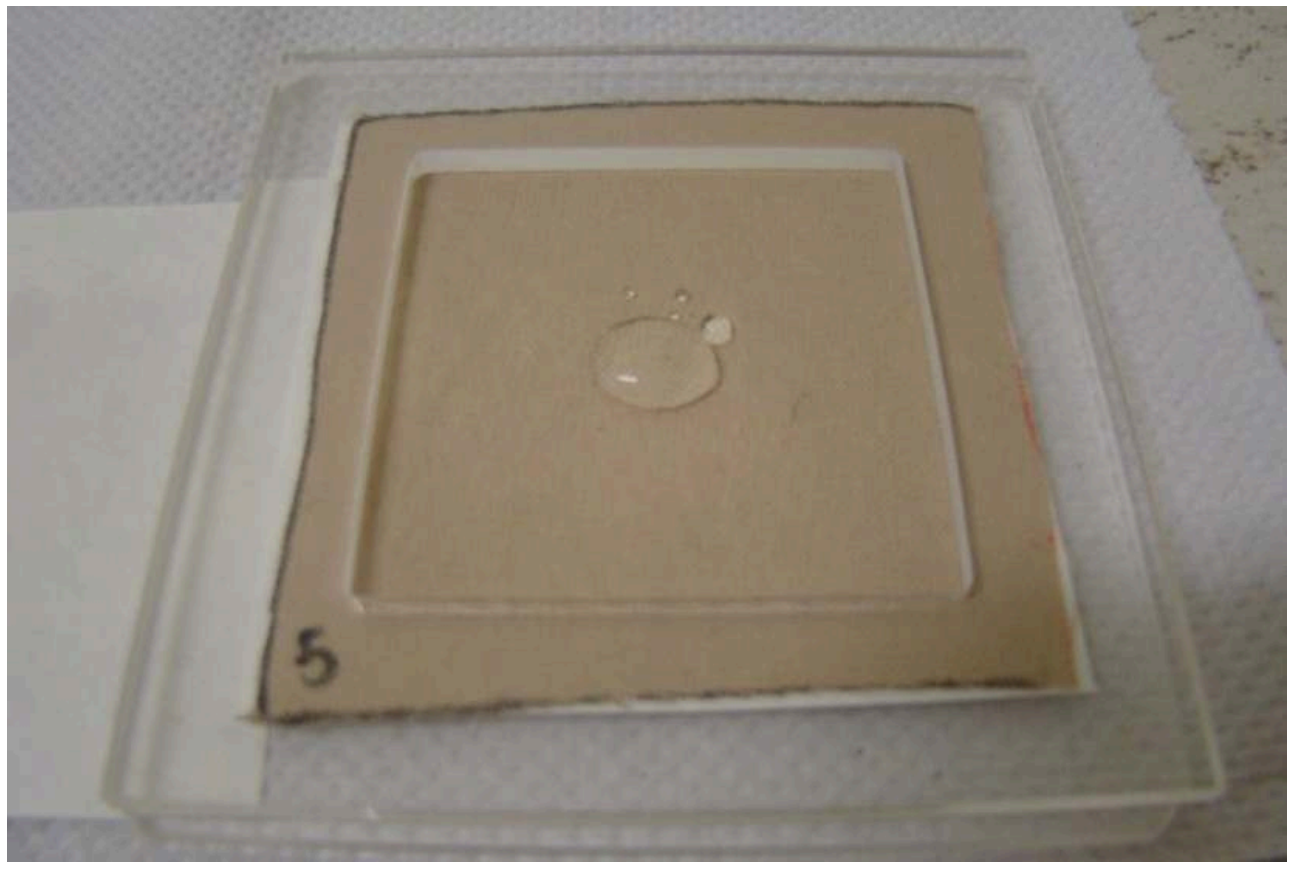

6 - Após a retirada da placa moldura, foram separadas as três camadas com o auxílio de uma pinça, manipulando-as pelas bordas ou por áreas que não estivessem contaminadas pela gota do agrotóxico e foram pesadas em balança de precisão de 0,0001g (Figura 5).

Figura 5. Separação das três camadas do teste; papel absorvente inferior (indicando penetração), papel absorvente superior (indicando repelência) e amostra do tecido (indicando retenção).

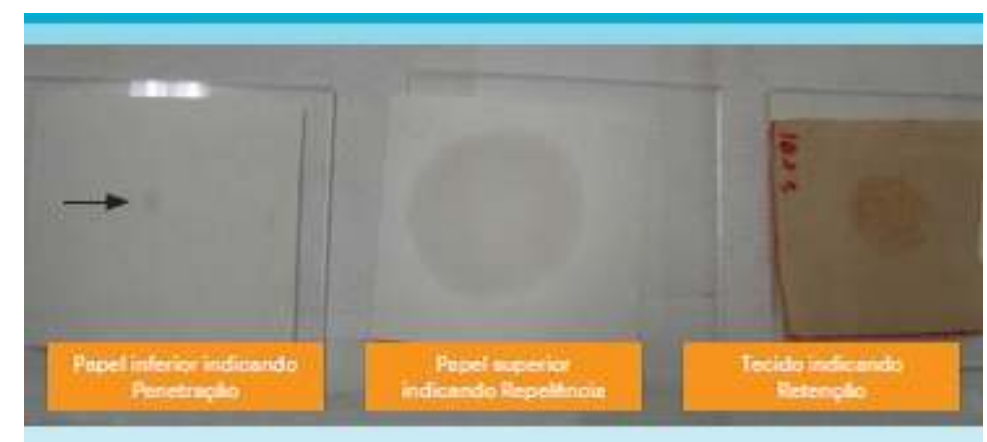

\section{Resultados}

A relação entre as variáveis dependentes contínuas (penetração, retenção e repelência) com a variável independente qualitativa nominal (tipo de material) está apresentada na Tabela 1 que mostra os valores da massa do papel de filtro e do material no início e ao final do experimento pelo tipo de material. Observou-se um peso médio de gota de $0,382 \mathrm{~g}$. 
Tabela 1. Valores da massa do papel de filtro e do material no início e no final do experimento pelo tipo de material (Tecido Simples (TS); Costura Simples (CS); Costura Rebatida (CR)).

\begin{tabular}{|c|c|c|c|c|c|c|c|c|c|c|}
\hline $\begin{array}{l}\text { Tipo } \\
\text { Material }\end{array}$ & $\begin{array}{l}\text { Superior } \\
(\mathrm{g}) \\
\text { Início }\end{array}$ & $\begin{array}{l}\text { Material } \\
(\mathrm{g}) \\
\text { Início }\end{array}$ & $\begin{array}{l}\text { Inferior } \\
(\mathrm{g}) \\
\text { Início }\end{array}$ & $\begin{array}{l}\text { Superior } \\
(\mathrm{g}) \\
\text { Final }\end{array}$ & $\begin{array}{l}\text { Material } \\
(\mathrm{g}) \\
\text { Final }\end{array}$ & $\begin{array}{l}\text { Inferior } \\
\text { (g) } \\
\text { Final }\end{array}$ & $\begin{array}{l}\text { Repelência } \\
(\%)\end{array}$ & $\begin{array}{l}\text { Retenção } \\
(\%)\end{array}$ & $\begin{array}{l}\text { Penetração } \\
(\%)\end{array}$ & $\begin{array}{l}\text { Massa } \\
\text { da } \\
\text { Gota } \\
\text { (g) }\end{array}$ \\
\hline TS & 0,941 & 0,788 & 0,923 & 1,299 & 0,796 & 0,933 & 95,461 & 2,029 & 2,510 & 0,375 \\
\hline TS & 0,947 & 0,794 & 0,933 & 1,314 & 0,813 & 0,943 & 92,722 & 4,903 & 2,376 & 0,396 \\
\hline TS & 0,920 & 0,760 & 0,976 & 1,287 & 0,768 & 0,985 & 95,596 & 2,111 & 2,293 & 0,384 \\
\hline TS & 0,941 & 0,767 & 0,908 & 1,299 & 0,776 & 0,917 & 95,513 & 2,163 & 2,324 & 0,374 \\
\hline TS & 0,926 & 0,775 & 0,985 & 1,295 & 0,782 & 0,993 & 95,893 & 1,898 & 2,210 & 0,385 \\
\hline TS & 0,882 & 0,769 & 0,880 & 1,234 & 0,778 & 0,887 & 95,636 & 2,413 & 1,952 & 0,369 \\
\hline TS & 0,927 & 0,781 & 0,926 & 1,298 & 0,790 & 0,935 & 95,469 & 2,240 & 2,291 & 0,388 \\
\hline TS & 0,926 & 0,752 & 0,982 & 1,282 & 0,761 & 0,991 & 95,418 & 2,304 & 2,278 & 0,373 \\
\hline TS & $*$ & $*$ & * & $*$ & $*$ & $*$ & $*$ & $*$ & $*$ & $*$ \\
\hline TS & 0,918 & 0,787 & 0,964 & 1,284 & 0,795 & 0,974 & 95,113 & 2,287 & 2,599 & 0,385 \\
\hline $\mathrm{CS}$ & 0,925 & 1,040 & 0,899 & 1,272 & 1,049 & 0,905 & 95,915 & 2,595 & 1,490 & 0,362 \\
\hline $\mathrm{CS}$ & 0,902 & 1,047 & 0,899 & 1,275 & 1,063 & 0,907 & 93,933 & 3,953 & 2,115 & 0,397 \\
\hline CS & 0,904 & 0,934 & 0,925 & 1,216 & 0,944 & 0,933 & 94,522 & 2,966 & 2,512 & 0,330 \\
\hline $\mathrm{CS}$ & 0,939 & 1,047 & 0,928 & 1,297 & 1,066 & 0,939 & 92,260 & 4,876 & 2,864 & 0,388 \\
\hline $\mathrm{CS}$ & 0,923 & 0,991 & 0,916 & 1,316 & 1,004 & 0,922 & 95,367 & 3,177 & 1,455 & 0,412 \\
\hline $\mathrm{CS}$ & 0,968 & 1,002 & 0,966 & 1,340 & 1,012 & 0,969 & 96,698 & 2,652 & 0,650 & 0,385 \\
\hline $\mathrm{CS}$ & 0,890 & 1,070 & 0,904 & 1,259 & 1,084 & 0,910 & 95,010 & 3,447 & 1,543 & 0,389 \\
\hline CS & 0,857 & 1,141 & 0,914 & 1,204 & 1,156 & 0,918 & 94,671 & 4,154 & 1,175 & 0,366 \\
\hline CS & 0,915 & 0,982 & 0,901 & 1,278 & 0,996 & 0,908 & 94,657 & 3,518 & 1,824 & 0,384 \\
\hline CS & 0,935 & 1,003 & 0,900 & 1,282 & 1,022 & 0,911 & 91,841 & 5,176 & 2,984 & 0,379 \\
\hline CR & 0,914 & 1,061 & 0,921 & 1,263 & 1,077 & 0,930 & 93,339 & 4,280 & 2,381 & 0,374 \\
\hline CR & 0,891 & 1,037 & 0,909 & 1,236 & 1,060 & 0,925 & 89,961 & 5,893 & 4,146 & 0,384 \\
\hline CR & 0,909 & 0,972 & 0,886 & 1,251 & 0,995 & 0,906 & 89,082 & 5,875 & 5,043 & 0,385 \\
\hline
\end{tabular}




\begin{tabular}{|l|l|l|l|l|l|l|l|l|l|l|}
\hline $\mathrm{CR}$ & 0,950 & 0,986 & 0,952 & 1,298 & 1,012 & 0,973 & 87,949 & 6,759 & 5,291 & 0,395 \\
\hline $\mathrm{CR}$ & 0,883 & 1,072 & 0,967 & 1,243 & 1,095 & 0,977 & 91,404 & 5,900 & 2,696 & 0,393 \\
\hline $\mathrm{CR}$ & 0,924 & 1,027 & 0,910 & 1,223 & 1,060 & 0,962 & 77,769 & 8,679 & 13,552 & 0,384 \\
\hline $\mathrm{CR}$ & $*$ & $*$ & $*$ & $*$ & $*$ & $*$ & $*$ & $*$ & $*$ & $*$ \\
\hline $\mathrm{CR}$ & 0,870 & 0,978 & 0,916 & 1,155 & 1,008 & 0,983 & 74,589 & 7,835 & 17,576 & 0,383 \\
\hline $\mathrm{CR}$ & 0,950 & 1,014 & 0,952 & 1,288 & 1,045 & 0,961 & 89,449 & 8,298 & 2,253 & 0,377 \\
\hline $\mathrm{CR}$ & 0,927 & 1,203 & 0,940 & 1,290 & 1,224 & 0,945 & 93,237 & 5,323 & 1,440 & 0,389 \\
\hline
\end{tabular}

$50 \quad(*)$ - Resultados retirados devido a problemas analíticos

51 A metodologia da gravimetria se mostrou de fácil execução, apesar de ser um procedimento trabalhoso quando se analisa muitas amostras.

52 O item 8.1.2 da norma ISO 22608/2004 permite o uso de praticamente qualquer líquido para a realização do teste, independente do grau de viscosidade. Isso possibilita o uso de líquidos com alta viscosidade, o que resultaria em baixa penetração (alta retenção), inviabilizando uma análise mais precisa da eficiência do equipamento de proteção. Esse aspecto pode ser considerado uma limitação do método.

53 Além disso, o teste da gravimetria considera que o valor médio de 10 amostras é suficiente para determinar a porcentagem da penetração, repelência e retenção, assumindo desnecessário assegurar estatisticamente que o valor encontrado encontrase dentro do limite esperado. Em amostras com grande variância, o valor médio pode não garantir a segurança e a eficiência do equipamento de proteção individual.

A partir da publicação da Portaria 189, de julho de 2010, estabeleceu-se que os níveis de proteção dos EPI, para o controle da exposição dérmica, deveriam atender, no mínimo, ao descrito no nível de desempenho $1 b$, o qual definia que a penetração da substância teste no material para confeccionar os EPI deve ser menor ou igual a $40 \%$, utilizando-se o procedimento da norma ISO 22608:2004. Entretanto, em 29 de junho de 2011, a Portaria 246 alterou a Portaria 189 e estabeleceu que os EPI devem, no mínimo, atender ao descrito no nível de desempenho dois da norma ISO 27065. O nível de desempenho dois determina a redução da porcentagem de penetração para menor ou igual a $5 \%$.

Constatou-se que a presença de costura afeta a permeabilidade (Tabela 2). A repelência é reduzida devido à presença de costura. A costura rebatida apresentou maiores porcentagens de penetração e de retenção; e menor de repelência. Observaram-se alterações na retenção nos EPI com costura simples. O tecido sem costura revelou possuir alta repelência e uma baixa retenção.

Essa análise mostra que uma perda significativa da repelência não indica necessariamente um ganho significativo na penetração, pois essa perda pode ser compensada pela elevação da retenção.

Tabela 2. Valores das médias, medianas e desvio padrão da massa do papel de filtro e do material no início e no final do experimento pelo tipo de material (Tecido Simples (TS); Costura Simples (CS); Costura Rebatida (CR)). 


\begin{tabular}{|l|l|l|l|l|l|l|l|l|l|l|}
\hline $\begin{array}{l}\text { Tipo } \\
\text { Material }\end{array}$ & $\begin{array}{l}\text { Superior } \\
\text { Início }\end{array}$ & $\begin{array}{l}\text { Material } \\
(\mathrm{g}) \\
\text { Início }\end{array}$ & $\begin{array}{l}\text { Inferior } \\
(\mathrm{g}) \\
\text { Início }\end{array}$ & $\begin{array}{l}\text { Superior } \\
(\mathrm{g}) \\
\text { Final }\end{array}$ & $\begin{array}{l}\text { Material } \\
(\mathrm{g}) \\
\text { Final }\end{array}$ & $\begin{array}{l}\text { Inferior } \\
(\mathrm{g}) \\
\text { Final }\end{array}$ & $\begin{array}{l}\text { Repelência } \\
(\%)\end{array}$ & $\begin{array}{l}\text { Retenção } \\
(\%)\end{array}$ & $\begin{array}{l}\text { Penetração } \\
(\%)\end{array}$ & $\begin{array}{l}\text { Massa } \\
\text { da } \\
\text { Gota } \\
(\mathrm{g})\end{array}$ \\
\hline TS & $\mathbf{0 , 9 2 5}$ & $\mathbf{0 , 7 7 5}$ & $\mathbf{0 , 9 4 2}$ & $\mathbf{1 , 2 8 8}$ & $\mathbf{0 , 7 8 4}$ & $\mathbf{0 , 9 5 1}$ & $\mathbf{9 5 , 2 0 2}$ & $\mathbf{2 , 4 8 3}$ & $\mathbf{2 , 3 1 5}$ & $\mathbf{0 , 3 8 1}$ \\
\hline SD & 0,019 & 0,014 & 0,037 & 0,022 & 0,016 & 0,037 & 0,953 & 0,920 & 0,183 & 0,009 \\
\hline MED & 0,926 & 0,775 & 0,933 & 1,295 & 0,782 & 0,943 & 95,469 & 2,240 & 2,293 & 0,384 \\
\hline CS & $\mathbf{0 , 9 1 6}$ & $\mathbf{1 , 0 2 6}$ & $\mathbf{0 , 9 1 5}$ & $\mathbf{1 , 2 7 4}$ & $\mathbf{1 , 0 4 0}$ & $\mathbf{0 , 9 2 2}$ & $\mathbf{9 4 , 4 8 7}$ & $\mathbf{3 , 6 5 1}$ & $\mathbf{1 , 8 6 1}$ & $\mathbf{0 , 3 7 9}$ \\
\hline SD & 0,030 & 0,057 & 0,021 & 0,041 & 0,058 & 0,020 & 1,502 & 0,884 & 0,752 & 0,022 \\
\hline MED & 0,919 & 1,021 & 0,909 & 1,276 & 1,036 & 0,915 & 94,664 & 3,482 & 1,684 & 0,384 \\
\hline CR & $\mathbf{0 , 9 1 3}$ & $\mathbf{1 , 0 3 9}$ & $\mathbf{0 , 9 2 8}$ & $\mathbf{1 , 2 5 0}$ & $\mathbf{1 , 0 6 4}$ & $\mathbf{0 , 9 5 1}$ & $\mathbf{8 7 , 4 2 0}$ & $\mathbf{6 , 5 3 8}$ & $\mathbf{6 , 0 4 2}$ & $\mathbf{0 , 3 8 5}$ \\
\hline SD & 0,028 & 0,071 & 0,026 & 0,044 & 0,069 & 0,027 & 6,672 & 1,468 & 5,643 & 0,007 \\
\hline MED & 0,914 & 1,027 & 0,921 & 1,251 & 1,060 & 0,961 & 89,449 & 5,900 & 4,146 & 0,384 \\
\hline
\end{tabular}

Os resultados encontrados foram significativos, com baixa variabilidade, comprovados pela proximidade entre média e mediana; e baixo valor do desvio padrão. A penetração é a variável que impacta diretamente na saúde do trabalhador. Quando ocorre a penetração, há uma exposição dérmica não controlada do trabalhador ao agrotóxico, sinalizando uma ineficiência do Equipamento de Proteção.

Os equipamentos de proteção estudados se classificavam como nível dois de repelência, conforme a norma ISO 22608 , necessitam ter repelência superior a $90 \%$ e penetração inferior a 5\%, em equipamentos de proteção novos.

60 Portanto, se fosse considerado o estabelecido na Portaria 189, de 22 julho de 2010, os materiais com tecidos simples e com costura simples atenderiam a norma. Contudo, os materiais com costura rebatida não atenderiam a norma.

61 Os resultados encontrados neste estudo são coerentes com resultados de estudos anteriores. Saleh, Kamel, El-Demerdash \& Jones (1998) também verificaram uma baixa penetração dos inseticidas propoxur, permethrin/pyrethrins e chlorpyrifos/allethrin em tecidos novos $100 \%$ de algodão. Os resultados encontrados por Shaw et al. (2001) também apresentaram alta repelência para atrazina, 0,23\%, em tecidos de algodão. Quando se avalia a presença e o tipo de costura, observou-se que o tecido sem costura foi o que apresentou a maior repelência, seguido pelo tecido com costura simples. 0 tecido com costura rebatida foi o que demostrou menor porcentagem de repelência. Em relação à retenção temos uma ordem inversa, sendo o tecido com a costura rebatida o que demonstrou a maior porcentagem de retenção.

63 Os resultados encontrados mostraram que uma perda na repelência pode ser compensada com um aumento na retenção, indicando que a permeabilidade do EPI (penetração) tem uma relação indireta com essas variáveis (repelência e retenção). 
Todos esses resultados podem subsidiar as definições dos padrões adequados a serem considerados na elaboração de normas sobre EPI. O estudo ainda sugere que os projetos de equipamentos de proteção tenham menor número de costuras, principalmente evitar costuras rebatidas.

\section{Conclusões e Recomendações}

5 Este estudo teve como objetivo investigar a eficiência dos equipamentos de proteção individuais utilizados por profissionais de Saúde Pública para evitar a exposição a agrotóxicos. Analisou-se o percentual de repelência, retenção e penetração dos equipamentos de proteção individual utilizados por profissionais de saúde no controle espacial de vetores, mosquito Aedes aegypti.

O estudo foi inovador ao comparar situação de campo com ensaios laboratoriais envolvendo EPI utilizados em saúde publica e analisar a viabilidade das normas pertinentes à avaliação dos equipamentos de proteção individual. Os resultados também contribuíram para ampliação dos conhecimentos sobre segurança no trabalho na utilização de agrotóxicos. É importante salientar que isso foi conseguido com base em uma metodologia que respeitou os preceitos da metodologia cientifica e da ética em pesquisa.

7 Como resultado adicional, foi observado que a presença de costuras afeta a penetração e a retenção de agrotóxicos sendo melhor para um design mais eficaz para o EPI diminuir a presença de costuras na vestimenta. A penetração é a variável mais importante na exposição dos trabalhadores. Contudo, não se pode negligenciar a variável retenção devido a possibilidade de contaminação dos trabalhadores ao vestir e ao despir os EPI já usados e impregnados com agrotóxicos devido a retenção.

ere-se que caso o produto utilizado nas análises fosse um químico mais viscoso, como Malathion diluído em óleo (produto utilizado no controle de vetores) ao invés do glifosato, os valores de retenção seriam bem superiores. Esse resultado é decorrente da imprecisão do método para análise de químicos mais viscosos, pela falta de capilaridade do material do filtro que poderia não absorver o químico, resultando em material repelido que poderia ser considerado como retido.

Uma vez que, os resultados variam com à solução empregada, sugere-se estudar as porcentagens de penetração, repelência e retenção em demais modelos de equipamentos de proteção com diferentes tecidos e com os demais agrotóxicos utilizados em saúde pública (alfa cipermetrina, deltametrina, fenitrotion, malathion, etc.).

Os resultados do estudo mostraram peculiaridades da legislação vigente que devem ser considerados. Os critérios para aprovação dos EPI segundo o método da gravimetria, adotados pela portaria 246, não considera a dosagem, a toxidade nem a exposição dérmica. Por isso, o critério de porcentagem de penetração é um critério incompleto para aprovação de um EPI como medida efetiva de proteção.

71 O teste da gravimetria considera que o valor médio de 10 amostras é suficiente para determinar a porcentagem da penetração, repelência e retenção, assumindo desnecessário assegurar estatisticamente que este valor encontra-se dentro de um limite. Nesse estudo, devido a pouca variabilidade dos resultados encontrados a média das 10 amostras foi um valor adequado. Contudo, em amostras com grande variância, o valor médio não garante segurança dos equipamentos de proteção individual. 
72 Esse estudo demonstrou que os equipamentos de proteção não tem cumprido sua função. Constatou-se que a presença de costura afeta a repelência. Foi observado um acréscimo na penetração devido a presença de costura, sendo a costura simples a com maior penetração. Observam-se também diferenças na retenção, sendo a costura rebatida a com maior porcentagem de retenção, seguida pela costura simples. o tecido liso revelou possuir uma baixa retenção.

73 Contudo, essa análise mostra que uma perda significativa da repelência não indica necessariamente um ganho significativo na penetração, pois essa perda pode ser atribuída à retenção.

74 O estudo restringiu-se a analise de EPI novos e traz a limitação de não avaliar a correlação entre a penetração, retenção e repelência com o desgaste associado ao uso. As condições reais de trabalho e de conservação dos EPI incorporam variáveis relevantes à saúde e à segurança dos trabalhadores. Porém, a análise de EPIs usados introduz complicações metodológicas para validação e para generalização dos resultados. Sugere-se pesquisas com análise de estudos de casos referente a permeabilidade de EPIs em situações reais de trabalho.

Identifica-se a pertinência da realização de estudos futuros que aprofundem e ampliem os conhecimentos sobre as exposições dérmicas as quais os trabalhadores que utilizam equipamentos de proteção na saúde pública estão vulneráveis, bem como os projetos e ergonomia na utilização desses EPI.

Sugere se, como temas a serem abordados em pesquisas futuras a interferência da gravidade e da capilaridade no método da pipeta; estudos com outros tecidos e não tecidos; a padronização do uso de um produto padrão (e.g. água destilada) no método da pipeta; e considerar parâmetros de conforto térmico nos equipamentos de proteção. Recomenda-se, ainda, pesquisas para ampliar os estudos dos EPI buscando correlação com outros parâmetros, como granulometria, número e tipos de lavagens.

\section{BIBLIOGRAFIA}

Abrasco - Associação Brasileira de Saúde Coletiva (2016). Nota técnica sobre microcefalia e doenças vetoriais relacionadas ao Aedes aegypti: os perigos das abordagens com larvicidas e nebulizações químicas - fumacê. Disponível em http://www.abrasco.org.br.

Baldi, I., Lebailly, P., Jean, S., Rougetet, L., Dulaurent, S., \& Marquet, P. (2006). Pesticide contamination of workers in vineyards in France. Journal of Exposure Analysis and Environmental Epidemiology, 16, 2, 115-124.

Blair, A., Sandler, D., Thomas, K., Hoppin, J., Kamel, F., Coble, J., Lee, W., Rusiecki, J., Knott, C., Dosemeci, M., Lynch, C.F., Lubin, J., \& Alavanja, M. (2005). Disease and Injury Among Participants in the Agricultural Health Study. Journal of Agricultural Safety and Health, 11, 2, 141-150.

Jain, R., \& Raheel, M. (2003). Barrier efficacy of woven and nonwoven fabrics used for protective clothing: predictive models. Bulletin Environmental Contamination and Toxicology, 71, 3, 437-446. 
Lee, S., \& Obendorf S.K. (2006). Developing protective textile materials as barriers to liquid penetration using melt-electrospinning. Journal of Applied Polymer Science, 102, 4, 3430-3437.

Machera, K., Goumenou, M., \& Glass, C.R. (2003). Determination of potencial dermal and inhalation operator exposure to malathion in grenhouses with the whole body dosimetry method. Annals of Occupational Hygiene, 47, 1, 61 -70.

Machera, K., Tsakirakis, A., Charistou,A., Anastasiadou, P., \& Glass, C. R (2009). Dermal Exposure of Pesticide Applicators as a Measure of Coverall Performance Under Field Conditions. Annals of Occupational Hygiene, 53, 6, 573-584.

Meirelles, L.A., Veiga M.M., \& Duarte, F.J.C.M. (2012). Efficiency of personal protective equipment used in agriculture. Work, 41, Suppl 1, 14-18.

MMA - Ministério do Meio Ambiente (2016). Agrotóxicos. Acessado em maio, 26, 2016, http:// www.mma.gov.br/seguranca-quimica/agrotoxicos.

OIT - Organização Internacional do Trabalho (2001). Guidelines on occupational safety and health management systems. Geneva: ILO-OSH 2001.

Raheel, M. (1988). Dermal exposure to pesticides: the barrier effectiveness of protective clothing. Journal of Environmental Health, 51, 2, 82-84.

Saleh, M. A., Kamel, A., El-Demerdash, A., \& Jones, J. (1998). Penetration of household insecticides through different types of textile fabrics. Chemosphere, 36, 7, 1543-1552.

Shaw, A., \& Hill, K. (1991). Effect of exposure time on the sorption of pesticide emulsifiable concentrates through microporous fabrics. Bulletin of Environmental Contamination and Toxicology, $46,45-52$.

Shaw, A., Cohen, E., \& Hinz, T. (2004). Laboratory Test Methods to Measure Repellency, Retention, and Penetration of Liquid Pesticides Through Protective Clothing Part II: Revision of Three Test Methods. Textile Research Journal, 78(8), 684-688.

Shaw, A., Cohen, E., Hinz, T., \& Herzig, B. (2001). Laboratory test methods to measure repellency, retention and penetration of liquid pesticides through protective clothing. Part I: comparison of three test methods. Textile Research Journal, 71, 10, 879-884.

Tácio, M. B.; Oliveira, M. L. De, \& Machado Neto, J. G. (2008). Eficiência de vestimentas hidrorrepelentes novas na proteção do tratorista em pulverizações de agrotóxicos em goiaba com o pulverizador de arrasto tratorizado de jato transportado. Revista Brasileira de Fruticultura, Jaboticabal, 30, 1, 106-111.

Van Hemmen, J. J. (1992). Agricultural pesticide exposure data bases for risk assessment. Reviews of Environmental Contamination and Toxicology, 126, 1-85.

Veiga, M. M. (2007). A contaminação por agrotóxicos e os Equipamentos de Proteção Individual (EPIs). Revista Brasileira de Saúde Ocupacional, 32 (116), 57-68.

WHO - World Health Organization (2005). Guidelines on Situation Analysis for Public Health Pesticide Management. Who/Cds/Whopes/Gcdpp/2005, 12.

Zhang, X., \& Raheel, M. (2003). Statistical model for predicting pesticide penetration in oven bricks used for chemical protective clothing. Bulletin of Environmental Contamination and Toxicology, $70,4,652-659$. 


\section{RESUMOS}

Os Equipamentos de Proteção Individuais (EPI) são utilizados na Saúde Pública para proteger os trabalhadores que utilizam agrotóxicos para controle de vetores de doenças. A exposição dérmica representa o maior risco de contaminação. A permeabilidade é uma característica que torna os EPI inadequados ao uso. Mesmo os EPI confeccionados com materiais impermeáveis, podem ser permeáveis, devido à porosidade dos tecidos e as costuras. Este estudo analisou a permeabilidade dos EPI aos agrotóxicos utilizados no combate ao mosquito Aedes Aegypti. A permeabilidade foi avaliada através do método da pipeta, calculando-se as porcentagens de repelência, de retenção e de penetração dos agrotóxicos nos EPI. Os resultados mostraram que: o tipo de costura influencia na permeabilidade; e que uma menor repelência pode não ser suficiente para avaliar a eficiência de um EPI, pois esta pode ser compensada por uma maior retenção, resultando em uma menor penetração.

Los Equipos de Protección Personal (EPP) son utilizados en la salud pública para proteger a los trabajadores que manejan plaguicidas para el control de vectores de enfermedades. La exposición cutánea es el mayor riesgo de contaminación. La permeabilidad de los EPP es una característica que hace su uso inadecuado. Incluso los EPP hechos de materiales impermeables pueden ser permeables debido a la porosidad de la tela y a las costuras. Esta investigación analizó la permeabilidad de los EPP a los plaguicidas utilizados para combatir el mosquito Aedes Aegypti. La permeabilidad se evaluó por el método de la pipeta, computando el porcentaje de repelencia, retención y penetración de los plaguicidas en los EPP. Los resultados mostraron que el tipo de costura influye en la permeabilidad y que una repelencia inferior puede no ser suficiente para evaluar la eficiencia de un EPP, ya que puede ser compensada por una mayor retención, lo que resulta en menos penetración.

Les équipements de protection individuelle (EPI) sont utilisés en santé publique pour protéger les travailleurs qui utilisent des pesticides pour lutter contre certaines maladies. L'exposition cutanée représente le plus grand risque de contamination. La perméabilité est une caractéristique des EPI qui les rend impropres à une utilisation. Même les EPI fabriqués à partir de matériaux imperméables sont perméables en raison de la porosité du tissu et des coutures. Cette étude a analysé la perméabilité des EPI contre les pesticides utilisés pour lutter contre les moustiques Aedes aegypti. La perméabilité a été évaluée par la méthode de la pipette, en calculant les pourcentages de répulsion, de rétention et de pénétration des pesticides dans l'EPI. Les résultats ont montré : que le type de couture influence la perméabilité; et qu'une répulsivité inférieure peut ne pas être suffisante pour évaluer l'efficacité de l'EPI, puisque celle-ci peut être compensée par une plus grande rétention, ce qui entraîne moins de pénétration.

Personal Protective Equipment (PPE) is used in Public Health to protect the workers who use pesticides for disease control. The dermal exposure is the highest contamination risk. The permeability is a characteristic that makes PPE unsuitable for using. Even those PPE which are made from waterproof materials, can be permeable, due to fabric porosity and the presence of seams. This study analysed the permeability to pesticides on PPE used by health personnel controlling Aedes aegypti mosquito. Permeability was evaluated through the pipette method, by calculating repellency, retention and penetration percentages of pesticides in the PPE. The results showed that the type of seam influences permeability; and a lower repellency may not be sufficient to evaluate the efficiency of PPE, as this can be compensated by a greater retention, resulting in less penetration. 
ÍNDICE

Mots-clés: équipement de protection individuelle; perméabilité; pesticides.

Palavras-chave: equipamento de proteção individual, permeabilidade, agrotóxico.

Keywords: personal protective equipment; permeability; pesticide

Palabras claves: equipo de protección personal; permeabilidad; plaguicida

\section{AUTORES}

\section{MARCELO MOTTA VEIGA}

Fundação Oswaldo Cruz, Ministério da Saúde, Rua Leopoldo Bulhões, 1480/503, CEP: 22.041-210, Rio de Janeiro, Brasil

Universidade Federal do Estado do Rio de Janeiro, Rua Voluntários da Pátria, 107, CEP: 22.20-000.

Rio de Janeiro, Brasil

marcelo.veiga@unirio.br

\section{CARLOS FREDERICO CAMPELO DE ALBUQUERQUE E MELO}

Organização Pan-Americana da Saúde / Organização Mundial da Saúde, Setor de Embaixadas Norte, Lote 19, CEP: 70800-400, Brasília, Brasil

meloc@paho.org 